\title{
Die Konzeptualisierung der Naturheilkunde im 19. Jahrhundert
}

\author{
(J.H. Rausse, Theodor Hahn, Lorenz Gleich) \\ Von Karl Eduard Rothschuh*
}

\section{Einleitung}

Die Naturheilverfahren mit der Anwendung von Wasser, Licht, Luft, Diät, Bewegung usw. sind innerhalb der Medizin seit den ältesten Zeiten im Gebrauch. Sie wurden früher theoretisch begründet und gelehrt als Wissenschaft von den 6 res non naturales (vgl. H. Schipperges 1962), als Lehre vom rechten Verhalten zur Erhaltung der Gesundheit, als Gesundheitsregiment. Speziell auf die Nutzung des kalten Wassers bezogen, gibt es eine wissenschaftliche Balneologie, die sich mit der Beschaffenheit, den Inhaltsstoffen der Wässer und der Theorie der Wasserwirkungen, zumal als Trank, beschäftigt, schon im 16. Jahrhundert (vgl. I. Probst 1971). Das Baden und Wassertrinken durch Gesunde und Kranke ist in gewissen Zeiten immer wieder sehr modern gewesen; es scheint vor allem in Zeiten des Reichtums und des Luxus gepflegt worden zu sein (vgl. A. Martin 1906). Eine solche Welle der Wasserheilkunst, begleitet von einer wahren Unzahl von balneologischen Schriften, zieht sich durch die erste Hälfte des 19. Jahrhunderts (vgl. B. Hirschel 1840). Sie steht stark unter dem Einfluß des «Naturismus» von Rousseau. Dieser Naturismus ist auch der weltanschauliche Hintergrund, der die Entwicklung von der Wasserheilkunde zur Naturheilbewegung katalysiert hat, wie ich es kürzlich darzustellen versucht habe (1981).

Die Naturheilbewegung, die sich durch das 19. Jahrhundert bis in unsere Zeit erstreckt und die Ende des vergangenen Jahrhunderts in die Lebensreformbewegung ausläuft, hat m.E. drei Anteile: 1. eine Grundidee von Weltanschauungscharakter, die ich als «Naturismus» bezeichnen möchte, welche aus einer Praxis eine «Bewegung» gemacht hat, 2. eine Bevorzugung «naturgemäßer», aus der unverfälschten Natur zur Verfügung stehender Heilanwendungen (Wasser, Licht, Luft, Diät usw.) = «Naturheilverfahren»

\footnotetext{
* Herrn Prof. Dr. Heinrich Buess zum 70. Geburtstag mit allen guten Wünschen gewidmet.
} 
und 3. eine Lehre, eine Kunde, eine Doktrin über Gesundheit und Krankheit, über ihre Quellen und die Wirkungsweise der Heilmittel = «Naturheilkunde». An der Ausbildung dieser drei Anteile der Naturheilbewegung haben Ärzte und Nichtärzte in recht verschiedenem Umfang mitgewirkt.

Hier soll über jene Versuche zur Konzeptualisierung der Naturheilideen in einer Theorie von Gesundheit, Krankheit und Heilung gesprochen werden, die auf dem Boden der Laienärzte, der sog. «Naturärzte», gewachsen ist. Diese Naturheilkunde entwickelte sich zur Zeit des «therapeutischen Nihilismus» (vgl. H. Buess 1947, Erna Lesky 1960) zu einer Kontrastmedizin gegen die «Medizinheilkunde» oder die wissenschaftliche «Schulmedizin» (vgl. A. Wölfing 1974). An diesem Kampf und an der Entwicklung einer Alternativdoktrin haben drei Männer besonderen Anteil gehabt, die in der offiziellen Medizingeschichtsschreibung bisher wenig Beachtung gefunden haben, während sie in der Selbstdarstellung der Naturheilbewegung ausführlich behandelt werden, etwa von Alfred Brauchle in der «Geschichte der Naturheilkunde in Lebensbildern» (1951) oder von Gerhard Hildebrand in der «Geschichte der Naturheilbewegung» (Berlin 1934). Die erwähnten drei Männer, die sich an der Ausbildung einer Lehre von der Naturheilung maßgeblich beteiligten, waren J.H. Rausse, Theodor Hahn und Lorenz Gleich. Von ihrem Anteil an der Konzeptualisierung soll hier die Rede sein. Alle drei nahmen ihren Weg aus der Wasserheilkunde des frühen 19. Jahrhunderts. Ihr Anteil am Ausbau der Naturheilpraxis steht hier nicht zur Debatte.

\section{J.H.Rausse (1805-1848). Biographisches - Ergographisches}

Rausse hat in Ernst Kapp einen liebevollen Biographen (1850) und in seiner Schwester eine gute Porträtistin gefunden, so daß wir über ihn relativ gut Bescheid wissen. Er wurde als Sohn des Superintendenten Franke zu Güstrow in Melckenburg als H. F. Franke 1805 geboren und nahm erst später den Schriftstellernamen J.H. Rausse an. Nach lustlosen Studien in der Theologie und einem liederlich-zügellosen Studentenleben (1824-28) entschloß er sich zur Forstlaufbahn in Aschaffenburg. Unter starkem Einfluß von Rousseau stehend, wollte er anschließend die Natur in ihrem Urzustand kennenlernen und lebte dazu $3 / 4 \mathrm{Jahr}$ in der Wildnis Nordamerikas unter den Osage-Indianern, bis ihn ein Gelbfieber gesundheitlich völlig zerrüttete und zur Rückkehr in die Heimat zwang (ca. 1834). Die Mußezeit benutzte er zu 
schriftstellerischen Versuchen, u. a. «Reisescenen aus zwei Welten» (1836). 1837 fuhr er zur Wiederherstellung seiner Gesundheit zu Vinzenz Prießnitz auf den Gräfenberg. Dort wurde er ein begeisterter Anhänger der Wasserkuren. Er wurde ein vielgelesener Autor von Schriften zur Wasserheilkunde, war später Leiter verschiedener Wasserheilstätten und starb mit 43 Jahren an einer Verengung des Magenausgangs, offensichtlich an Krebs (A. Brauchle 1951, S. 123). Rausse hat sich sehr um die Theorie und Praxis der Wasserheilkunde bemüht. Von ihm gehen die ersten Versuche von Laien aus, die Wirkungen «natürlicher» Heilanwendungen durch eine Theorie, durch ein Alternativkonzept zur wissenschaftlichen Medizin zu erklären und danach die Indikationen aufzustellen.

Seine wichtigsten Schriften sind 1. «Der Gräfenberger Wasserarzt» (1838), 2. Auflage 1840 (hier abgekürzt mit Grä.); 2. «Miscellen zur Gräfenberger Wasserkur» (1839), 2. Auflage 1840 (abgekürzt Mis.); 3. «Über die gewöhnlichsten ärztlichen Mißgriffe beim Gebrauch des Wassers als Heilmittel» (1847) (abgekürzt ä.M.) und 4. «Anleitung zur Ausübung der Wasserheilkunde» (1850), wovon nur die erste Abteilung noch von Rausse selbst stammt, während die zweite und dritte Abteilung von Rausses Schüler Theodor Hahn nach Rausses Tod geschrieben worden sind (abgekürzt R.H. = Rausse-Hahn).

Weder Rausse noch Gleich noch Theodor Hahn werden im «Biographischen Lexikon der hervorragenden Ärzte ...» (2. Aufl.) genannt. Rausses Schriften stehen aber in Callisen, Medicinisches Schriftstellerlexikon, Bd.31, 1843, ferner im Index Medicus. Man kann daraus entnehmen, daß seine Miscellen 1842 ins Schwedische, 1848 ins Englische übersetzt wurden. Das Buch über die ärztlichen Mißgriffe (1847) wurde 1855 ins Englische übersetzt.

\section{Rausses Beiträge zur Theorie der Naturheilkunde}

Rausses erste Wasserschrift, der Gräfenberger Wasserarzt, ist noch sehr gemäßigt im Ton, im Stil, in den Urteilen; sie ist eine Apologie der Heilkräfte des Vinzenz Prießnitz in Gräfenberg und als Wegweiser für interessierte Kranke gedacht. In der Erklärung, wie das Wasser heilend wirke, hält er sich ganz an die Darstellung von Johann Siegmund Hahn junior (1696-1773/S. 5, 16) in seiner klassischen Schrift «Unterricht von der wunderbaren Heilkraft des frischen Wassers» (1743). Sie wurde im frühen 19. Jahrhundert von dem 
Schulmann und Wasserpropagandisten Eucharius Ferdinand Christian Oertel (1765-1850) in mehreren Auflagen wieder herausgebracht. «Wasser» - so sagt Rausse mit Hahn (Grä.1840, S.11) - «ist unter allen flüssigen Körpern am geschicktesten, sich in die feinsten Äderchen, Fäserchen und Nerven des menschlichen Leibes, ja gar in die kaum durchs Vergrößerungsglas sichtbaren Gefäßchen, Fibern der Haare, Nägel und Knochen hineinzubegeben und darin zu bewegen.» Wasser wirkt, so meint Rausse weiter, auflösend und zerteilend; es nimmt den scharfen Säften ihre Kraft. Seine Frische wirkt belebend, erquickend und stärkend (S.11). Das kalte Wasser wirkt ferner, so heißt es später (S.50/51) reinigend, verdünnend, fördert die Ausscheidung der scharfen und galligen Stoffe; es ist das beste urin- und schweißtreibende Mittel, verdünnt die stockenden Säfte, fördert die Verdauung und den Blutumlauf. Das alles sind Begriffe entweder der Iatromechaniker aus der Schule von Friedrich Hoffmann oder aus der bis ins 19. Jahrhundert nachwirkenden Humoralpathologie. Viel Eigenes, Originelles ist in dem ersten Büchlein Rausses zur Theorie nicht zu finden.

Das ist völlig anders in seinem nächsten Buch, den «Miscellen». Es beginnt mit dem überheblichen Satz (Vorrede zur 1. Auflage): «Wahrscheinlich werden diese Blätter nicht ohne Irrthümer in Nebensachen sein; aber in der Hauptsache enthält dies Buch mehr Wahrheiten als zusammengenommen die sämmtlichen medicinischen Schriften und Systeme von Galen an bis auf die neueste Zeit - was freilich nicht viel sagen will.» Hier beginnt eine von Affekten gespeiste Angriffsschrift gegen die «Allopathie» und die «Medizinheilkunde». Was uns daran interessiert, ist Rausses Idee und Konzept von Krankheit und Heilung. Es läßt sich in folgenden Sätzen zusammenfassen: Der Organismus unterliegt der Notwendigkeit, fortgesetzt fremde Substanzen zu assimilieren und sie dazu zu zersetzen (1840, S.29). Krankheitsursachen sind stets materiale Stoffe (S.33); sie bilden sich entweder innen aus einer unzureichenden Ausscheidung oder stammen von außen in Gestalt von «Giften». Die abnormen Anstrengungen, um diese Krankheitsstoffe hinaus zu stoßen, sind die Symptome der akuten Krankheiten (S. 38). Krankheit ist also eine Anstrengung, um Schädliches auszuführen, und die akute Krankheit ist demnach heilsam (S.213). Ihre Symptome dürfen nicht «durch Medizin oder Blutzapfen» unterdrückt werden (S.45). Chronische Leiden lassen sich nur beseitigen, wenn sie durch $\mathrm{W}$ asseranwendungen wieder in akute Krankheiten zurückverwandelt werden (S.214). Viel Trinken von kaltem Wasser ruft im Magen reaktive Wärme hervor und fördert die Zersetzung der Stoffe. Die Haut ist ein Ausscheidungsorgan, das durch das 
kalte Wasser zur verstärkten Reaktion gebracht wird (S.112). Die ersten Ursachen von Krankheiten sind also Stockungen von Stoffen im Körper (S.119). Fieber und Entzündung sind heilsam (S.124ff.). Fieber ist also keine Krankheit (S.219). Das Fieber weicht, wenn die Auswerfung der schädlichen Stoffe abgeschlossen ist. «Äußere Geschwüre und Ausschläge» werden dadurch erzeugt, daß der Körper fremde Stoffe und Gifte aus seinem Inneren «auf die Haut wirft» (S. 146); diese Erscheinungen müssen gefördert und nicht unterdrückt werden. Die Wasserkur erreicht ihr Ziel über Krisen, die sich im Auftreten von stinkendem Schweiß (S.340), von Geschwüren und Ausschlägen äußern (S.199ff.). Austreibung der Krankheitsstoffe durch Luft und Wasser, «das ist die Behandlung, welche die Natur jedem ihrer Geschöpfe angedeihen läßt. Ohne Wasserdiät von innen und außen ist keine lebenslängliche Gesundheit möglich. Nicht vor uns in grauen Mysterien und Doctrinen der Zukunft liegt die Heilkunst, sondern weit hinter uns in der Vergangenheit des grünen Naturlebens. ¿Zurück〉 heißt das Motto der wahren Heilkunst, nicht 〈Vorwärts〉!» (S.217).

Das Buch ist durchsetzt von maßlosen Angriffen gegen die «Medizinheilkunde» oder «Medizinkunst» (S.222). Die Ärzte treiben Medizinvergiftung (S.224ff.) mit Arsenik, Mercur, Blausäure, Belladonna und anderen höllischen Giftessenzen (S.256). Das Publikum ist durch Medizinärzte umstrickt (S. 261). Aber die Menschen verfügen über einen Instinkt, der es ihnen gestattet, «das Zuträgliche für Gesundheit und Leben vom Schädlichen zu unterscheiden» (S.311). Er ist untrüglich. Das meiste von dem, was Rausse hier ausspricht, hat er wohl bei Prießnitz beobachtet, der es aber selbst nicht formulieren konnte (Vorwort 1.Aufl.).

Als Ergänzung zu den Miscellen veröffentlichte Rausse 1847 das Buch «Über die gewöhnlichsten ärztlichen Mißgriffe ...». Darin findet sich eine besondere Abhandlung «Ueber die Aufsaugung und Ablagerung der Gifte und Medikamente im lebenden animalischen Körper» (S.136-199). Hier bespricht er mit Literaturzitaten die Ergebnisse wissenschaftlicher chemischer Untersuchungen, welche das Vorkommen vieler «Gifte» im Blut, Harn usw. beweisen. Da Rausse solche Schriften nicht zu lesen pflegte, hat er sie entweder aus einer Übersicht, wahrscheinlich aus Oesterlens Heilmittelkunde (1844), entnommen oder sich von einem kenntnisreichen Mitarbeiter-ich denke, es war Theodor Hahn-zusammenstellen lassen. Es tauchen berühmte Autoren jener Jahre auf, Tiedemann, Gmelin, Magendie, Rutherford, Wöhler, Runge, Liebig und viele andere. Alles ist bestückt mit heftigen Angriffen auf die «Pillenjesuiten» in der Medizin. 
In seinem letzten Buch «Anleitung zur Ausübung der Wasserheilkunde», das Theodor Hahn aus dem Nachlaß von Rausse zusammenstellte, fügte Rausse dem Bisherigen einen fortwirkenden neuen Gedanken hinzu (Abt. I, S. 1 ff.). Die Heilkunde darf sich keiner Krankheitsklassifikation bedienen, denn in der Natur gibt es keine Genera und Species, sondern nur Individuen. Ihre Einteilung in Gattungen und Arten darf der Pathologe vornehmen; aber in der Heilkunst ist sie unanwendbar, «weil nothwendig die Heilkunst auf einer Basis beruhen muß, die jeden Irrthum und Fehlgriff unmöglich macht ... Diese Basis nun ist keine andere, als der Inbegriff der sinnlich wahrnehmbaren Krankheitszeichen, und zwar nur derjenigen Krankheitszeichen, die so deutlich und so markirt sind, daß bei ihrer Auffassung kein Mensch irren kann, der seine gesunden fünf Sinne hat» (S.30). «Wenn man die Wasseranwendung nach Krankheitszeichen lehrt, so lehrt man damit auch die Kunst des Individualisirens» (S.32). Aus diesen Grundsätzen ist das später in der Naturheilbewegung immer wieder als Schlachtruf geäuBerte Postulat entstanden: «Nicht Krankheiten, sondern Kranke behandeln!» So steht es etwa bei Schweninger (1906).

Alle diese Ideen wären nicht möglich gewesen ohne die Überzeugung von der absoluten Zweckmäßigkeit des Leibes. «Jede vitale Function des lebenden Organismus (auch die abnormste, d.i. krankste) ist die denkbar zweckmäßigste bei den jedesmaligen inneren chemischen und StructurVerhältnissen des Körpers» (ä.M., S. 18), und dieser Glaube stützt sich auf ein weltanschaulich getragenes Urvertrauen in die Natur. Man muß nur nach ihr leben: «O die große liebende Natur hat dem Menschen wie dem Thier keinen Trieb gegeben, dessen Befriedigung unglücklich oder ungesund macht» (Misc., S. 117). «Was durch die Natur ist, das ist gut» (Misc., S. 27), wie es schon Rousseau verkündet hat. Das bestätigt sich für Rausse durch das, was Reisende über die «körperliche Vollkommenheit der meisten Wilden» berichten (Misc., S.317). Rausses Naturismus schimmert überall durch, wenn man darauf achtet.

\section{Zur Würdigung von Rausse}

Es ist nicht zu bezweifeln, daß Rausse die wesentlichen Gedanken jener Theorie der Krankheit und Heilung entwickelt hat, die seitdem die Vorstellungswelt der Anhänger der Naturheilkunde beherrscht hat. Wir werden alles wiederfinden, z. B. in den Äußerungen von Alfred Brauchle im Ge- 
spräch mit L.R.Grote (1935). Einige wesentliche Gedanken hat noch Rausses Schüler Theodor Hahn beigesteuert. Davon soll weiter unten die Rede sein.

Rausse war ein seltsames Gemisch von Künstler, Apostel, Naturarzt, von Ignoranz und Arroganz. Er war von leidenschaftlichem Temperament und schoß oft weit über das Ziel hinaus, vor allem in der Verurteilung seiner Gegner. Er war ungeheuer erfindungsreich in herabsetzenden Schimpfworten für die wissenschaftlichen Mediziner, die Pillenjesuiten und Giftmischer. Wahr ist, daß seitdem die Atmosphäre zwischen «Schulmedizin» und «Naturheilkunde» schwer getrübt ist. Beide Worte kommen übrigens bei Rausse noch nicht vor; sie entstanden später (vgl. A. Wölfing 1974). Natürlich wurde auch Rausse seinerseits heftig angegriffen. Sein Freund und Schüler Hahn hat sich sehr bemüht, ihn zu verteidigen (R.-H. 2. Abt., S. 228-251).

Rausse konnte recht flüssig schreiben, und das schriftstellerische Talent tritt überall in seinen Schriften zutage. Aber das machte seine Texte auch vielfach überlastet mit Einfällen. Er war kein wissenschaftlich-systematischer Denker. Die Schlüssigkeit seiner Meinungen als ein Ganzes ist erst nach mehrmaligem Lesen erkennbar. Er war auch kein Mann der Wissenschaft; seine Urteile über Chemie (ä.M., S.145) oder Medizin sind oft recht wirr. Rausse selbst hat sicher wenig medizinische Schriften studiert. Er nennt nicht einmal Hufeland, der ihm weltanschaulich und gedanklich doch nahestand und bei dem er manchen hilfreichen Gedanken für sein Konzept hätte finden können. Er war ungeheuer vital, arrogant und überheblich. Das war wohl seine Natur; nicht umsonst nannten ihn seine Kommilitonen auf der Forstakademie «Roß»; davon leitet sich übrigens das Pseudonym «Rausse» ab (vgl. E. Kapp 1850). Brauchle feiert Rausse als Philosophen und genialen Denker der Wasserheilkunde (1951, S. 124), aber sieht auch seine «dämonische Natur». Sein Verhalten grenzte manchmal an Manie. Er schreibt auf das Titelblatt der Miscellen 1840 unter seinen Autorennamen: «wirklicher geheimer Zauberer und großer Medicus beim Stamm der Schlangenindianer, correspondierendes Mitglied aller Akademien und gelehrten Gesellschaften in den Ländern der Karaiben und Hottentotten, Ritter unzählig vieler Orden aus den Staaten Lichtenhain, Ziegenhain und Passendorf». Da spricht sich ein Gemisch von Arroganz und Selbstironie aus, das schwer durchschaubar ist. Bernhard Hirschel (1815-1874) hat in seiner «Hydriatica» 1840 Rausse als geisteskrank eingestuft (S.189), an fixem Wahn leidend usw. Das ist sicher übertrieben. So schwankt sein Bild in den 
Augen von Freund und Feind. Zu seiner Entschuldigung kann man anführen, daß die Medizin seiner Zeit tatsächlich viele Angriffspunkte bot, wobei die heroischen Aderlässe und Arzneimittelgaben nur die eine Seite darstellen.

Was ihm bleibt, ist die Konzeption wesentlicher Grundsätze der späteren Naturheilkunde. Zur Praxis des Wasserheilverfahrens hat er viele glückliche Ideen und Indikationen beigesteuert. Das ist aber hier nicht unser Thema. Über Rausses schriftstellerisch-belletristischen Werke kann hier kein Urteil abgegeben werden. Diese Bücher waren nicht erhältlich.

Theodor Hahn (1824-1883) als Fortsetzer der theoretischen Bemühungen von Rausse

Theodor Hahn hat Rausse menschlich und sachlich sehr nahegestanden. Er war von Hause Apotheker, in Chemie, Heilmittellehre und medizinischer Literatur wohlbewandert. Er litt seit Kinderzeiten an schwerem Asthma und wurde von Rausse geheilt und zur Wasserheilkunde geführt. Er war überdies ein Vetter von Rausse. Er kam als Kranker am 1. Oktober 1847 in die Wasserheilanstalt Rausses in Lehsen in Mecklenburg und blieb seitdem als Mitarbeiter bei ihm. Als Rausse Ostern 1848 die Wasserheilstätte Alexandersbad übernahm, ging Hahn mit ihm und blieb dort über den Tod Rausses (am 12. Juli 1848) hinaus. Hahn dürfte maßgeblichen Anteil an der sehr sachverständigen Studie «Ueber die Aufsaugung und Ablagerung der Gifte und Medikamente im lebenden animalischen Körper» (in ä. M. 1847, S.134-199) haben, die eine bedeutende Literaturkenntnis beweist, die Rausse nicht hatte. Auch hatte Hahn ein ruhigeres Temperament und eine mildere Feder. Beide haben sich gut ergänzt. Hahn übernahm es dann, die unveröffentlichten Nachlaßmanuskripte von Rausse zu ordnen und herauszugeben. Die daraus geschaffene «Anleitung zur Ausübung der Wasserheilkunde für Jedermann, der zu lesen versteht» umfaßt drei Abteilungen. Davon stammt die erste (1850) gänzlich von Rausse; sie enthält überwiegend praktische Ratschläge zur Durchführung von Wasseranwendungen. Die zweite Abteilung (II) bricht nach 7 Seiten ab. «Soweit der verewigte Rausse», schreibt Hahn. Alles Weitere ist Hahnsche Bearbeitung. Er bringt dabei einen neuen wichtigen Gedanken ins Spiel. Auf den Organismus in seinen Wandlungsprozeß wirken unausgesetzt Lebensreize ein, z. B. Speise und Trank, Luft, Wärme, Licht, Elektrizität, Bewegung und Ruhe (S.17). 
Wirken normale Lebensreize ein, so bleibt der Mensch gesund; wirken abnorme Reize ein, wird er krank. Mit dem Reizbegriff schließt sich Hahn enger an die damals in der Medizin gängigen Ideen von John Brown und Hufeland an (vgl. Rothschuh 1978). Als abnorme Reize stehen die materiellen ganz im Vordergrund (das sind Rausses «Stoffe»); sie können äußerlich z. B. alimentarische oder giftige Stoffe, auch Kontagien und Miasmen sein oder aber im Körper durch Entziehung der normalen Lebensreize entstehen und in ihm zurückgehalten werden. In den übrigen Punkten folgt Hahn der Darstellung Rausses. Krankheiten können geheilt werden durch Herstellung der Einwirkung normaler Lebensweise und Aufhebung der Einwirkung abnormer Lebensreize. Das sind die Heilmittel der «Naturheilkunde». Das Wort taucht hier bei Hahn (1851, S. 24) erstmals auf, nicht bei Rausse; aber geprägt hat es wohl Lorenz Gleich (1849) in einem Vortrag vom 29. Juli 1849 in München. «Alle schädlichen Einflüsse, also alle abnormen Lebensreize sind zugleich Heilmittel», meint Hahn weiter. Medikamente sind keine Heilmittel (S. 25/26). In der Naturheilkunde kennt Hahn nur zwei wesentliche akute Krankheitszeichen, Entzündung und Fieber (II/28). Er bestätigt Rausses Krankheits- und Heilungslehre, die Stofflichkeit der Krankheitsursachen, die Heilung durch Ausscheidung dieser Stoffe (II/57). In keiner Krankheit sollte ein Medikament eingenommen werden. Es gibt lokal kühlende, erregende und ableitende Verfahren (II/77). Die Ausscheidung des materiellen Krankheitsstoffes erfolgt in einer Krisis. In der Abteilung III (erschienen 1852) werden die chronischen Krankheitszeichen und ihre Behandlung geschildert. Hahn spricht jetzt konsequent von «Naturheilkunde». Ihr oberster Grundsatz lautet: «Gemäß der Natur heilen», d.h. gemäß der Natur des kranken Organismus, gemäß seiner «Lebenskraft» (III/6). Hahn ordnet das ganze Erfahrungsgut nicht nach Krankheitsnamen, sondern nach Krankheitszeichen, als Erschlaffung oder Verstärkung. «Nicht ein einziges Krankheitszeichen ist zu trennen von dem Gesamtbegriff Lebenskraft ...» (III/40). Im Jahre 1852 begann Hahn fleischlos zu leben und wurde Vegetarier. Er hat nach Brauchle die vegetarische Ernährung als dem Wasser ebenbürtige Behandlung in die Naturheilkunde eingeführt. Sein «Praktisches Handbuch der naturgemäßen Heilweise» hat seit 1865 viele Auflagen erlebt; es enthält eine Gesundheitslehre und eine Krankheitslehre mit sehr ausführlichen Angaben über Nahrung, Speise, Trank usw. In der Krankheitslehre wird jetzt allerdings wieder nach den wissenschaftlichen Namen der Krankheiten, nicht mehr nach den Krankheitssymptomen gegliedert, wie er es noch 1852 (s. o.) gemacht hatte. Hahn war ein tüchtiger 
Mann, aber auch nicht ohne Selbstbewußtsein. Im Vorwort zur zweiten Auflage des Handbuchs (1867) heißt es von dem Buch: «Ich bin sowohl der Diät, wie der Heilweise der Zukunft weit, weit, nicht bloß um Jahrzehnte, vielleicht selbst um Jahrhunderte voraus.»

Mit Theodor Hahn ist wohl die Theorie der Naturheilkunde praktisch vollendet. Zur Prägung der Begriffe Naturarzt, Naturheilkunde, naturgemäße Lebensweise, Naturheilweise, hat wohl Dr.Lorenz Gleich (17981865), ein bayerischer Militärarzt, das Maßgebliche beigetragen.

\section{Die Theorie der Naturheilkunde bis auf unsere Zeit}

Es ist bemerkenswert, daß sich in den Grundgedanken der Theorie der Naturheilkunde von der zweiten Hälfte des 19. Jahrhunderts nichts Wesentliches mehr verändert hat. Es sind einige neue Gesichtspunkte hinzugekommen, etwa die Rohkost, die Mineralstofflehre, die Heilerde usw.; aber an den Grundsätzen hat sich nichts geändert. Dr. med. Franz Kleinschrod, Kurarzt in Wörishofen, veröffentlichte 1911 eine «Wissenschaftliche Begründung der Naturheilkunde». Er arbeitet die Unterschiede zwischen Naturheilkunde und der «Medizin der Schule» besonders scharf heraus und gibt von der Naturheilkunde folgende Definition (S. 109):

«Die Naturheilmethode ist ein Heilverfahren, welches theoretisch sich auf eine vitalistische und teleologische Auffassung vom Wesen der Krankheit stützt, in der Krankheit und im Symptom auch den Heilungs- und Regulationsvorgang erblickt, und praktisch in diesem Sinne die Krankheit zu behandeln sucht 1. kausal-symptomatisch durch Behandlung des Symptomes im Sinne der Entfernung und Ausscheidung der materiellen Krankheitsstoffe durch den naturgemäßen Verlauf der Krankheit (Entfernung der äußeren Krankheitsursache), 2.aktiv-vital (physikalisch-diätetisch) durch das Prinzip der aktiven Selbststärkung der Funktion und der Organe durch ihren natürlichen Gebrauch (physikalisch) und durch die richtige Ernährung (diätetisch) zur Entfernung der inneren Krankheitsursache, und welches sich dabei der natürlichen Mittel der Selbsterhaltung des Lebens bedient.

Dagegen lautet die Definition der medizinischen Schule, soweit deren Heilverfahren ein medikamentös-allopathisches und operatives ist: Die Schulmedizin ist ein Heilverfahren, welches sich theoretisch auf eine mechanistische Auffassung vom Wesen der Krankheit stützt, in der Krankheit und im Symptom nur eine pathologische mechanische Störung sieht, und praktisch in diesem Sinn die Krankheit und das Symptom zu behandeln sucht, 1. mechanisch-symptomatisch durch mechanische Unterdrückung oder Bekämpfung des Symptoms oder durch mechanische Zerstörung der Krankheitsursache oder des Krankheitsherdes, 2. mechanisch-funktionell durch eine Reizung der Funktion, durch spezifi- 
sche Reizmittel die Funktion mechanisch zu stärken sucht, und welche sich zu diesem Zweck der Wirkung von Medikamenten oder mechanischer, operativer Eingriffe bedient.»

In seiner Broschüre «20 Jahre im Dienste der Naturheilkunde. Betrachtungen und Belehrungen über Medizin und Naturheilkunde» (Baden-Baden 1911) werden von Maximilian Malten die gleichen Grundsätze vorgetragen. «Fort mit aller Medizin, zurück zur Natur» (S.40) ist der leitende Grundsatz.

Bis in die neueste Zeit reicht die praktische und schriftstellerische Tätigkeit von Alfred Brauchle (1898-1964). Er hat sich an vielen Stellen zur Naturheillehre geäußert, vielleicht am ausführlichsten in Alfred Brauchle und L. R. Grote: Gespräche über Schulmedizin und Naturheilkunde, Leipzig 1935. Seine Thesen lassen sich folgendermaßen in aller Kürze zusammenfassen: Die Naturheilkunde sucht über die Allgemeinbehandlung die Gesundheit zu erhalten und Kranke zu heilen (S. 13). Umschriebene Organerkrankungen gibt es nicht (S. 14). Die bedeutendsten Heilmöglichkeiten enthalten die natürlichen Reize wie Luft, Licht, Wasser, Bewegung, Atmung, Ernährung (S.15). Krankheit ist eine Abwehrleistung, Leiden eine gefahrvolle Schwäche (S.18). Der Natur entnommene Lebensreize sind wirksamer als chemische Arzneimittel (S.25). Die Naturheilkunde unterstützt die Symptome in ihrer Heilbestrebung (S.36). Man sollte weder Medikamente, noch Laboratorien, noch Tierversuche, noch das Spezialistentum überschätzen (S.39). Beachte den Instinkt des Kranken für das, was ihm zuträglich ist (S.52). Die Naturheilkunde hat stets von mangelhafter Ausscheidung gesprochen (S.59). Instinkt und Erfahrung sind die Hauptträger der naturheilerischen Behandlung (S.63). Der Naturheilkunde ist eine Verbindung von Diagnose und Therapie oft vollkommen gleichgültig (S. 71). Der Naturarzt hat Vertrauen zu dem inneren Arzt, der Heilkraft (S. 75). Was den Gesunden gesund erhält, genügt auch zur Gesundung des Kranken (S.93). Insgesamt kehren hier alle Thesen, Maximen und Lehren wieder, die schon bei Rausse und Hahn ihre Festlegung erfahren haben.

\section{Dr. Lorenz Gleich (1798-1865)}

Es gab um die Mitte des 19. Jahrhunderts zahlreiche Verfechter der praktischen Wasserheilkunde. Die Theoretiker waren immer in der Minderzahl. Unter ihnen erwarb sich Dr. Lorenz Gleich, ein bayerischer Militärarzt, besondere Verdienste um die Schaffung einer neuen Nomenklatur. Ein Sammelband: Dr. Gleich's physiatrische Schriften (1849-1858), München 
1860, enthält Vorträge und Aufsätze, von denen uns hier einige interessieren. In seinem Vortrag von Juli 1849 fordert er als Wasserheilkundiger, «Krankheiten unmöglich zu machen durch vollkommen naturgemäße Lebensweise» (S.3) und jegliche Krankheit nur durch die dem Körper innewohnende Lebenskraft heilen zu lassen. Gleich wird auch nicht müde, auf die Berücksichtigung des «Naturinstinktes» bei den Kranken hinzuweisen, vor allem in dem Aufsatz «Gibt es eine Naturheilkunde?» (München 1858). Hier heißt es: «Die Grundlage der ursprünglichen Heilkunde bliebe demnach kein gelehrtes, sondern das vom allmächtigen Schöpfer selbst geschaffene Heilsystem, dessen Seele war der Naturinstinkt, dadurch wurde es erst zum Naturheilsystem für Alles, was Leben hatte» (S.5). Die Heilverfahren durch «naturinstinktgemässe Heilmethoden» sind so alt wie das Menschengeschlecht selbst (S.6). Wie Gerhard Hildebrand (1934, S. 50 ff.) gezeigt hat, war durch die Existenz zweier sich bewährender, aber völlig verschiedener Natur-Regimina bei Prießnitz und Schroth neben der Anwendung von Wasser auch die Rolle der Ernährungsweise als natürliches Heilverfahren anerkannt. Man konnte also bei der Bezeichnung Hydrotherapie nicht stehenbleiben. Gleich hat bei Prießnitz in Gräfenberg und bei Johann Schroth in Lindewiese die Verfahren und ihre Erfolge studiert. An beiden fand er etwas Richtiges. Gleich machte auch Versuche mit vegetarischer Diät. Um die Einheit der Prinzipien bei unterschiedlichen Verfahren in einem Oberbegriff festzuhalten, schuf er die Begriffe Naturheilverfahren und Naturheilkunde. Die alte Bezeichnung «Hydropathie» für Wasserheilkunde sei falsch und lächerlich. Überhaupt sei die Wasserheilkunde als Name für das ganze Heilverfahren und ihre Vertreter unzureichend. Richtiger wäre «Naturheilkunde, Naturheilkunst, Naturheilverfahren, Naturheilmethode, physiatrisches Heilverfahren, Naturheilanstalt, Naturheilarzt oder Naturarzt» (1849, S.7). Die Naturheilkunde enthält drei Anteile, nämlich a) die Naturinstinktlehre, b) die Naturdiätetik und c) das Naturheilverfahren. Die Naturheilkunde ist identisch mit Physiatrie (S.8). Die Naturinstinktlehre äußert sich beim Gesunden und Kranken im Verlangen nach Ruhe, Getränk, Abkühlung, stärkenden Speisen oder Nahrungsenthaltung. «Alle sogenannten Wilden erfahren durch ihren Naturinstinkt, was Gelehrte in Büchern zusammengestellt haben» (S. 15). Naturdiätetik bedeutet naturinstinktgemäße Leibesbehandlung mit Diät, Bewegung, Bädern, Kleidung, Wasser, Luft usw. Naturheilverfahren heißt Heilen ohne Arzneistoffe und Blutentziehung, mit Kälte und Wärme, Trinken von kaltem Wasser, Umschlägen, Diät, frischer Luft usw. (S.18). Arzneimittel sollten nie angewendet werden. 
Gegen die neuen Bezeichnungen besteht nach Gleich (1856, S. 16) eine merkwürdige Abneigung, aber sie seien treffender. Die Notwendigkeit einer Reform der Hydropathie hat schon Rausse angebahnt; man sollte aber weiterhin neue Wege gehen. Das gilt u. a. für die Bezeichnungen; am besten ist Physiatrik oder Naturheilkunde. Die Wasserheilanstalten müssen also in «Naturheilanstalten» umgetauft werden (S. 18/19).

Prießnitz und Schroth haben zu den Naturheilbehandlungen Bewundernswertes beigetragen. Alle Arzneien, seien es allopathische oder homöopathische, bleiben vom Naturheilverfahren ausgeschlossen (nach Gleich, S.50). Der Naturarzt benutzt Mittel, welche die Lebenstätigkeiten stärken. «Diese Eigenheit der Naturarzneimittel aufzuspüren - das Verhalten dieser eigenthümlichen Kraft auf den gesunden und kranken Organismus auszuforschen, mit einem Worte: diese dynamischen nichtgiftigen Agentien der Naturarzneimittel zum Heilwerke dem Instinkt gemäß zu verwenden - das ist die Aufgabe und der Zweck der Naturheilkunde - Physiatrie - und das ausschließliche Heilverfahren mit diesen dynamischen nicht giftigen Naturheilagentien bei internen dynamischen Krankheiten der Unterschied des Naturarztes von Ärzten, die anderen Heilsystemen anhängen» (S.21). Eigenartigerweise wird Hufeland gar nicht erwähnt.

Gleichs Schriften kreisen also vor allem um eine bessere Terminologie. Der Ausdruck Naturheilverfahren und Physiatrie ist durch ihn allgemein bekannt gemacht worden. In seinen Unterscheidungen der Naturinstinktlehre, Naturheilkunde, Naturheilverfahren hat er den verschiedenen Anteilen in den Naturheilbestrebungen verbesserten Ausdruck verschafft. In der schroffen Ablehnung einer jeden Arznei ist er ein unerbittlicher Vorkämpfer dieser Grundhaltung, die sich jahrzehntelang durch die Naturheilkunde hindurch verfolgen läßt. Gleich hat also zum Selbstverständnis der Naturheilkunde Wesentliches beigetragen. Seitdem sind immer neue «Lebensreize» in die Naturheilkunde aufgenommen worden, wie die Gymnastik, die Luftbäder, die Lichtbehandlung, die Rohkost und vieles andere. Einiges davon ist alt und wurde schon vor oder außerhalb von Naturheilkunde ins Leben gerufen und gefordert; Weiteres entstand als neuartiges Verfahren innerhalb der Naturheilkunde. Jedenfalls erlaubte die Darlegung allgemeiner Prinzipien, über die Wasseranwendung hinaus den Weg zu einer allgemeineren Methodik naturheilerischer Verfahren und zu größerem Reichtum an Behandlungsarten zu beschreiten. Diese Entwicklung durchzieht die mittleren Jahrzehnte des 19. Jahrhunderts in Deutschland, Sie geht im letzten Viertel des Jahrhunderts in die Lebensreform-Bewegung über. 
In der Mischung aus irrationalem Naturismus, mit ihrem rationalisierten Kern und ihrer Fülle von Heilanwendungen lebt die Naturheilbewegung weiterhin fort. Sie durchwandert dabei ein kulturelles, zeitlich sich wandelndes Feld, das mit vielen Berührungsflächen das Selbstverständnis und die Praxis der Naturheilbewegung bereichert oder auch bedroht hat. 


\section{Literatur}

Brauchle, Alfred, und L.R.Grote, Gespräche über Schulmedizin und Naturheilkunde, Leipzig 1935.

Brauchle, Alfred, Die Geschichte der Naturheilkunde in Lebensbildern. Zweite erweiterte Aufl. von «Große Naturärzte», Stuttgart 1951. - 4.Auflage mit W.Groh: Zur Geschichte der Physiotherapie, Heidelberg 1971.

Buess, Heinrich, Zur Geschichte der Hydrotherapie. Ciba Z.9 (1947) 3919-3926.

Gleich, Lorenz, Physiatrische Schriften (1849-1858), München 1860. (Enthält Vorträge und Aufsätze zum Thema und Begriff Naturheilkunde.)

Hahn, Johann Siegmund, Unterricht von der wunderbaren Heilkraft des frischen Wassers (1743), hrsg. von Euch. Ferd. Christian Oertel, 5. Auflage, Ilmenau 1833.

Hahn, Theodor, Praktisches Handbuch der naturgemäßen Heilweise (1.Aufl. 1865), 4.Aufl., Berlin 1875.

Hildebrand, Gerhard, Zur Geschichte der Naturheilbewegung (bis zur Gründung des «Deutschen Bundes») in: Festschrift zum 45jährigen Bestehen des Deutschen Bundes der Vereine für naturgemäße Lebens- und Heilweise (Naturheilkunde) E. V., Berlin 1934.

Hirschel, Bernhard, Hydriatica oder Begründung der Wasserheilkunde auf wissenschaftliche Prinzipien, Geschichte und Literatur, Leipzig 1840.

Kapp, Ernst, J.H.Rausse, der Reformator der Wasserheilkunde, Hamburg 1850.

Lesky, Erna, Von den Ursprüngen des therapeutischen Nihilismus. Sudhoffs Archiv 44 (1960) $1-20$.

Martin, Alfred, Deutsches Badewesen in vergangenen Tagen, Jena 1906.

Oesterlen, Friedrich, Handbuch der Heilmittellehre, 1. Aufl., Tübingen 1844.

Probst, Irmgard, Die Balneologie des 16. Jahrhunderts im Spiegel der deutschen Badeschriften, Diss. med. Münster 1971.

Rausse, J. H., Reisescenen aus zwei Welten, 1836.

ders., Der Gräfenberger Wasserarzt ..., (anonym) Meissen (1838); 2. Aufl. 1840.

ders., Wasser thuts freilich! Miscellen zur Gräfenberger Wasserkur. 2 Theile. 2. Aufl., Zeitz 1840. ders., Über die gewöhnlichsten ärztlichen Mißgriffe beim Gebrauch des Wassers als Heilmittel ... nebst einer Abhandlung über die Aufsaugung und Ablagerung der Gifte und Medikamente im lebenden animalischen Körper, Zeitz 1847.

ders., Anleitung zur Ausübung der Wasserheilkunde. 3 Abteilungen. Leipzig 1850, 1851, 1852, hrsg. von Theodor Hahn.

Rothschuh, Karl Eduard, Konzepte der Medizin in Vergangenheit und Gegenwart, Stuttgart 1978.

ders., Über den Naturismus «als die weltanschauliche Grundlage der Naturheilbewegung». Z.f. Allgemeinmedizin 1981, im Druck.

Schweninger, Ernst, Der Arzt, Frankfurt a. M. 1906.

Wölfing, Achim, Entstehung und Bedeutung des Begriffes Schulmedizin. Die Auseinandersetzungen zwischen der naturwissenschaftlichen Medizin und Vertretern anderer Heilmethoden im 19. und 20. Jahrhundert. Diss. med. Freiburg 1974. 


\section{Summary}

In the first half of the 19th century a trend to hydrotherapy gave birth to a rich balneologic literature. One bathed and drank water. Three pioneers of this speciality are described. J.H. Rausse (1805-1848) stood under the influence of Rousseau and Prießnitz. He was in charge of hydropathic sanatoriums, wrote books and attacked the representatives of classical medicine: These Jesuits of pills were poisoning the patients with medicaments. After his theory, diseases are caused by material matters. Their expulsion, usually produced by fever, sweat, and rash, can be supported by water cures. Theodor Hahn (1824-1883), a cousin of Rausse, continued these efforts. He, too, regarded illness as a defence reaction or a process of regulation. The third man, Dr.Lorenz Gleich (1798-1865), was a Bavarian medical officer. Because the natural healing instinct of the body could not only be supported by water, but also by diet, he wrote that one should not speak of hydropathy. Instead of it he proposed the words Naturheilkunde or physiatry.

Prof. Dr.med. Karl Eduard Rothschuh

Waldeyerstraße 27

D-44 Münster/Westfalen 